Robles Rodríguez, A.; Abad Robles, M.T.; Robles Rodríguez, J. y Giménez Fuentes-Guerra, F.J. (2019) Factores que influyen en el proceso de formación de los judokas olímpicos / Factors That Influence the Process of Formation of the Olympic Judokas. Revista Internacional de Medicina y Ciencias de la Actividad Física y el Deporte vol. 19 (74) pp. 259-276 Http://cdeporte.rediris.es/revista/revista74/artfactores1022.htm

DOI: http://doi.org/10.15366/rimcafd2019.74.006

\title{
ORIGINAL
}

\section{FACTORES QUE INFLUYEN EN EL PROCESO DE FORMACIÓN DE LOS JUDOKAS OLÍMPICOS}

\section{FACTORS THAT INFLUENCE THE PROCESS OF FORMATION OF THE OLYMPIC JUDOKAS}

\author{
Robles Rodríguez, A. ${ }^{1}$; Abad Robles, M.T. ${ }^{2}$; Robles Rodríguez, J.. ${ }^{2}$ y \\ Giménez Fuentes-Guerra, F.J.2 \\ ${ }^{1}$ Centro Universitario San Isidoro. Adscrito a la Universidad Pablo de Olavide. Sevilla (España) \\ arobles@centrosanisidoro.es \\ 2 Universidad de Huelva. Facultad de Ciencias de la Educación (España) \\ manuel.abad@dempc.uhu.es, jose.robles@dempc.uhu.es, jfuentes@uhu.es
}

Código UNESCO / UNESCO Code: 6303 Sociología General / General Sociology.

Clasificación Consejo de Europa / Council of Europe Classification: 16 Sociología del Deporte / Sociology of Sport.

Recibido 22 de junio de 2017 Received June 22, 2017

Aceptado 15 de febrero de 2018 Accepted February 15, 2018

\section{RESUMEN}

Con el objetivo de analizar los factores que influyen en el proceso de formación deportiva de los judokas españoles olímpicos, se aplicó una entrevista semiestructurada de 52 preguntas agrupadas en 6 dimensiones (Contexto Deportivo, Contexto Social, Proceso de Formación, Psicológica, Técnico-táctica, Condición Física). Los resultados muestran que los judokas olímpicos se iniciaron en la práctica del Judo a los 6,90 $\pm 3,02$ años, concretamente las mujeres a los $6,80 \pm 2,88$ años y los hombres algo más tarde, a los $7 \pm 3,42$ años, el $95 \%(n=19)$ influenciados, fundamentalmente, por un familiar. Además, destaca que ninguno de los entrevistados consiguió medalla en categorías inferiores. Para el $75 \%(n=15)$ de los judokas olímpicos es muy importante llevar una sincronización del entrenamiento técnico-táctico, de la condición física y de los aspectos psicológicos de forma integrada.

PALABRAS CLAVES: Judo, Entrevista, Formación Deportiva, Juegos Olímpicos. 


\begin{abstract}
With the aim of analizing the factors that have an influence on the sports training process of the olympic Spanish judokas, a semistructured interview of 52 questions grouped in 6 aspects was carried out: Sports Context, Social Context, Training Process, Psychological Condition, Technical-Tactical Condition and Physical Conditon). The results show that olympic judokas began practising judo at the age of $6.90 \pm 3.02$ years. Specifically, women started at the age of $6.80 \pm 2.88$, and men slightly later, at the age of $7 \pm 3.42$ years. $95 \%$ $(n=19)$ of them were mainly influenced by a relative. Moreover, it is to be highlighted that none of the interviewees won a medal in low categories. For $75 \%(n=15)$ of the olympic judokas, it is very important that technical-tactical training, physical condition training and psychological aspects be synchronised in an integrated way.
\end{abstract}

KEY WORDS: Judo, Interview, Sports Formation, Olympic Games.

\title{
INTRODUCCIÓN
}

Desde finales del siglo XX, son numerosas las investigaciones que se han hecho eco de que llegar a la élite deportiva depende de multitud de aspectos, y no sólo de la cantidad de horas que dedica el deportista a entrenarse. En este sentido, se deben también considerar factores de tipo biológico, psicológico, perceptivo-cognitivo y social en continua interacción (Singer y Janelle, 1999). Por un lado, la preocupación por intentar comprender mejor los elementos que influyen en los deportistas para que éstos lleguen al máximo rendimiento deportivo y, por otro lado, el hecho de que con frecuencia encontremos que muchos de los jóvenes que presentan características deportivas propias de los talentos deportivos no lleguen a la excelencia deportiva ha propiciado un cambio de perspectiva en la detección de talentos. Según Lorenzo y Calleja (2010) la mayoría de los planteamientos en la detección de talentos han tomado como referencia la necesidad de ciertas aptitudes e intentan detectarlas lo antes posible en jóvenes. Esta forma clásica de detección de talentos va de arribaabajo (top-down) y no parece haberse mostrado muy eficaz. En este sentido, estamos de acuerdo con el planteamiento manifestado por Lorenzo (2001), para quien la selección de talentos tradicional centrada sólo en el control de los mejores se ha demostrado claramente ineficaz en los últimos años. Por otro lado, existe un enfoque de indagación que va de abajo-arriba (bottom-up) cuyo objetivo es analizar el proceso de formación de deportistas de alto rendimiento con la finalidad de encontrar unas condiciones óptimas de desarrollo de la pericia que se puedan aplicar a los nuevos valores (Regnier, Salmela y Russell, 1993).

Coincidiendo con Ruiz y Salinero (2011), en la actualidad el deportista y su rendimiento no pueden ser entendidos sin una concepción integral de él mismo y de su entorno, por lo que el éxito deportivo de éste difícilmente puede percibirse como un logro exclusivamente personal. Este entorno estará determinado, como hemos dicho, por multitud de elementos, entre los que destacan el entrenador (Giménez, 2003), la familia (García-Moya, Moreno, 
Rivera, Ramos y Jiménez-Iglesias, 2011) y los iguales (Lorenzo y Sampaio, 2005). También sobresalen los factores genéticos (Baker, Horton, RobertsonWilson y Wall, 2003) y los aspectos psicológicos (Mujika, 2010). El conocimiento, el estudio y la investigación de todas estas variables presentadas puede contribuir a que los deportistas tengan un proceso de formación más integral y riguroso, además de optimizar su rendimiento aumentando así las posibilidades de conseguir mejores resultados deportivos (Gimeno, Buceta y Pérez-Llantada, 2007). En este sentido, en la literatura especializada, nos encontramos con investigaciones sobre las características de la personalidad de los judokas para la predicción del rendimiento (Ruiz, 2008), trabajos que evalúan y realizan una intervención psicológica en talentos deportivos de Judo (Abalde y Pino, 2016; Gimeno y Guedea, 2001), estudios que analizan la relación entre judoka y entrenador (Ruiz, 2007), investigaciones que tratan de determinar los aspectos de la condición física más relevantes en los judokas de elite (Franchini, Del Vecchio, Ferreira, y Candau, 2015; Pion et al., 2015) y la importancia de la formación técnico-táctica de los deportistas (Osipov et al., 2016). Por otro lado, también existen estudios que analizan la opinión de los entrenadores de Judokas de elite en relación con la dirección del entrenamiento y la competición (Santos, Fernández-Río, Almansba, Sterkowicz y Callan, 2015) y con el entrenamiento psicológico (Sava y Ciuntea, 2013). Teniendo en cuenta los trabajos mencionados anteriormente, abogamos por el estudio y análisis del proceso de formación de los deportistas con el fin de conocer aquellos elementos que en mayor medida inciden en su excelencia deportiva a lo largo de toda su carrera, y así tenerlos en cuenta en la formación de futuros deportistas de elite. En este sentido, se estudiará la influencia de los contextos deportivo y social, el proceso de formación llevado a cabo, los aspectos psicológicos, técnico-tácticos y de la condición física. Estos factores han sido puestos de manifiesto en la literatura científica (Gimeno, Buceta y Pérez-Llantada, 2007), aunque se detecta un vacío respecto a estudios en los que se investigue sobre cómo ha sido el proceso de formación deportiva de los judokas que llegan al alto rendimiento, así como la influencia de los diferentes entornos a lo largo de su vida deportiva desde la perspectiva de los propios deportistas, lo cual puede resultar esclarecedor. No obstante, estudios de esta índole han sido realizados en deportes como el fútbol (Pazo, 2010), el baloncesto (Sáenz-López et al. 2006) y el atletismo (Palao, Ortega, Calderón, y Abraldes, 2008). Por estos motivos, el objetivo de esta investigación fue analizar los factores que influyen en el proceso de formación deportiva de los judokas españoles que habían representado a España en los Juegos Olímpicos celebrados en el siglo XXI: Sidney 2000, Atenas 2004, Pekín 2008, Londres 2012 y Río de Janeiro 2016.

\section{MATERIAL Y MÉTODO}

\section{Participantes}

En la investigación intervienen los judokas de elite españoles que han participado, al menos, una vez en unos Juegos Olímpicos en el Siglo XXI (Tabla 1). 
Tabla 1. Juegos Olímpicos y participantes.

\begin{tabular}{lccc}
\hline Juegos Olímpicos & $\begin{array}{c}\text { Participantes } \\
\text { femeninos }\end{array}$ & $\begin{array}{c}\text { Participantes } \\
\text { masculinos }\end{array}$ & $\begin{array}{c}\text { Número total de } \\
\text { participantes }\end{array}$ \\
\hline Sídney 2000 & 6 & 5 & 11 \\
Atenas 2004 & 4 & 6 & 10 \\
Pekín 2008 & 4 & 2 & 6 \\
Londres 2012 & 4 & 2 & 6 \\
Río de Janeiro 2016 & 3 & 2 & 5 \\
\hline
\end{tabular}

Se debe tener en cuenta que un mismo deportista puede haber participado en más de unos Juegos Olímpicos. En total en los Juegos Olímpicos celebrados en el siglo XXI han participado 25 judokas (15 mujeres y 10 hombres). La muestra final de entrevistados fue de 20 judokas (80\%): 12 mujeres (60\%) y 8 hombres (40\%).

\section{Instrumento}

Para la recogida de datos, teniendo en cuenta las características del estudio, hemos utilizado una metodología cualitativa de investigación (Anguera, 2008) con el fin de conseguir una descripción profunda del contexto de investigación, máxima objetividad, y una correcta recogida de datos que den lugar a la obtención de conocimiento con suficiente capacidad explicativa y acorde al objetivo planteado. Como instrumento de investigación se utilizó la entrevista semiestructurada diseñada y validada por Robles et al. (2016), la cual consta de 52 preguntas agrupadas en 6 dimensiones:

Contexto Deportivo. Encaminada al conocimiento de cómo fueron los inicios en el judo del deportista, qué le llevó a dedicarse a la alta competición, y si en sus inicios compartió la práctica del judo con la de otros deportes.

Contexto Social. Se pretende estudiar la influencia que ha podido tener en la formación deportiva y en el rendimiento del deportista su entorno más cercano (familiares, entrenadores, amigos...).

Proceso de Formación. Queremos conocer qué tipo de metodología de entrenamiento han seguido los judokas durante todo su proceso de formación deportiva, desde que edad y por qué motivos compiten, a qué se deben los éxitos conseguidos durante la carrera deportiva de los judokas, y por qué muchas jóvenes promesas se pierden y no llegan a la elite.

Psicológica. Se trata de conocer si el deportista ha trabajado con psicólogos, en caso positivo, de qué forma lo ha hecho, y, por último, a qué aspectos psicológicos da el deportista mayor importancia.

Técnico-táctica. Destinada a saber qué importancia les da el deportista a estos aspectos, si considera que los ha trabajado en su justa medida durante su formación deportiva y en qué etapa considera necesario profundizar más en ellos. 
Condición Física. Se procura conocer cómo la han entrenado, quién ha sido el responsable en su preparación, qué capacidades físicas consideran más importantes y cómo creen que se debería entrenar durante el proceso de formación deportiva.

En este trabajo nos centraremos en los aspectos más destacados de cada una de las dimensiones estudiadas.

\section{Procedimiento}

En primer lugar, se consultó la base de datos de la Real Federación Española de Judo y Deportes Asociados (RFEJYDA), donde se verificó el número de judokas españoles que habían sido olímpicos durante el siglo XXI, siendo la población total de 25 sujetos. Posteriormente, nos pusimos en contacto con los responsables de la RFEJYDA para obtener de forma confidencial los datos de contacto con los judokas olímpicos. Una vez preparada la entrevista y conseguidos los datos de los judokas olímpicos, nos pusimos en contacto con cada uno de ellos. Inicialmente este contacto fue vía email, a través de una carta donde se le informaba del estudio y en la que se le pedía la participación en el mismo. Tras la aceptación de cada uno de los judokas (20) se pasó al contacto telefónico para concertar el día y la hora en la que le realizaríamos la entrevista. Por último, hubo un tercer contacto telefónico con cada uno de ellos, en el que se llevó a cabo la entrevista propiamente dicha. Los entrevistados fueron informados sobre el objetivo del estudio y se les garantizó la confidencialidad de la información recogida bajo la Ley Orgánica 15/1999, de 13 de diciembre, sobre la Protección de Datos de Carácter Personal.

Las entrevistas fueron transcritas literalmente y analizadas con la ayuda del programa informático especializado para la investigación cualitativa MAXQDA 10. Para que el proceso de análisis fuese más fiable, la codificación se llevó a cabo por un grupo de 4 codificadores. Este grupo estaba compuesto por expertos en el campo de la codificación de entrevistas, al haber trabajado con este instrumento de investigación tanto en sus respectivas Tesis Doctorales como en otras investigaciones. La fiabilidad interna entre codificadores fue estimada por medio del acuerdo interobservador (AIO) (Thomas y Nelson, 2007), alcanzándose una fiabilidad interobservador óptima (86,76\%) (Goetz y LeCompte, 1984), lo que permitió que los miembros del grupo pudiesen codificar las entrevistas de manera individual. Además, para paliar los acuerdos debidos exclusivamente al azar, se calculó, utilizando el programa informático SPSS 18.0, el Índice de Kappa (Cohen, 1960), cuyo valor fue de 0,833 con $p<0,001$, considerado casi perfecto (Landis y Koch, 1977). Finalmente, con el objetivo de obtener mayor validez, todas las entrevistas de la investigación fueron repartidas entre los miembros del grupo de codificadores, de manera que cada entrevista fue analizada y codificada por dos personas. 


\section{RESULTADOS}

\section{Dimensión Contexto Deportivo}

Con esta dimensión se pretendía conocer los inicios del deportista en el Judo, razones que le llevaron a dedicarse a la alta competición, y saber si en sus inicios compartió la práctica del Judo con la de otros deportes. Los judokas Olímpicos se iniciaron en la práctica del Judo a los 6,90 $\pm 3,02$ años, concretamente las mujeres a los $6,80 \pm 2,88$ años y los hombres algo más tarde, a los $7 \pm 3,42$ años. Los principales motivos a los que aluden respecto al porqué empezaron a practicar Judo son: por la influencia directa de algún familiar $(n=7)$, por el influjo de los iguales $(n=3)$, por recomendaciones del profesor de educación física $(n=3)$ y por comenzar a realizar un deporte que les sirviera como defensa personal $(n=1)$. Respecto a las características que confieren los judokas a sus distintos entrenadores durante su proceso de formación deportiva, éstos resaltaron los siguientes: saber motivar en todo momento a los deportistas $(n=9)$, tener buen conocimiento de la disciplina deportiva $(n=4)$ y alentar e impulsar al deportista hacia la elite deportiva $(n=3)$.

Mis entrenadores son los que me han impulsado y han confiado en mí para llegar a donde podía llegar, (Sujeto 3, párrafo 14). Mi entrenador fue quien hizo que me gustara este deporte, y quien hizo que encontrara mi sitio y me encontrara bien dentro del tatami (Sujeto 17, párrafo 15).

\section{Dimensión Contexto Social}

En esta dimensión se pretendió analizar la influencia que ha podido tener, en la formación deportiva y en el rendimiento del deportista, su entorno más cercano (familiares, entrenadores, amigos...). En cuanto al entorno próximo los judokas Olímpicos, el $95 \%(n=19)$ señaló que durante el proceso de formación deportiva habían recibido gran apoyo familiar, fundamentalmente por parte de sus padres y hermanos, estando también presente sus entrenadores y amigos, aunque en menor medida, lo cual también ocurre una vez que se encuentran en la elite deportiva.

Pues lo más importante, porque mis padres siempre me han apoyado, siempre me han llevado a las competiciones que tenía que ir, y en la medida de lo posible me compraban todo lo que necesitaba para practicar judo (Sujeto 14, párrafo 14). El entorno social tiene mucha importancia. Si mi familia o mi pareja no me hubieran respetado, la vida de alta competición me habría costado más, es muy importante para rendir al máximo (Sujeto 17, párrafo 27).

El 55\% ( $n=11)$ de los judokas entrevistados reconocieron que el hecho de haber tenido un familiar directo, que había practicado con éxito Judo u otro deporte en alta competición, le había influido positivamente en su proceso de formación para llegar a la elite. Respecto a la importancia que otorgan a su 
formación académica, el 90\% ( $n=18)$ de los jukokas afirmaron que sus familias daban mucha importancia a los estudios. Además, el 100\% $(n=20)$ de los sujetos reconocen que es imprescindible compaginar los estudios con el Judo, principalmente para estar mejor situado laboralmente una vez terminada la alta competición $(n=18)$. Por otro lado, a pesar de dedicar gran parte de su vida al deporte, el $95 \%(n=19)$ de los entrevistados consideraron que las relaciones sociales con los demás fueron de vital importancia. Entre las principales razones, los judokas estimaron como las más relevantes, el hecho de desconectar y despejar la mente $(n=9)$, así como sentirse arropado y apoyado por su gente $(n=7)$.

Pienso que es importante, porque a veces es bueno romper con el judo, todo el día pensando en judo y deporte... creo que las relaciones sociales te ayudan a despejarte y desconectar un poco, que también es bueno (Sujeto 8, párrafo 23).

En cuanto a la presión del entorno, sólo el 30\% (n=6) de los judokas la sintieron. De ellos, la mayoría $(n=4)$ consideraban esta presión como positiva. Además, el $100 \%(n=20)$ de los judokas estimaron que para reducir esta presión sería conveniente contar con la ayuda de un psicólogo deportivo.

\section{Dimensión Proceso de Formación}

En esta dimensión se procuró analizar el recorrido deportivo de los judokas olímpicos desde que se iniciaron, a qué se debían, según su opinión, sus éxitos, por qué no llegaban muchas jóvenes promesas a la elite. En este sentido, ninguno de los entrevistados consiguió medalla a nivel nacional en las categorías más pequeñas, es decir, antes de los 14-15 años. El 60\% de los judokas comenzaron sus logros deportivos nacionales alrededor de los 15-16 años. Los éxitos deportivos se debían fundamentalmente a aspectos psicológicos como la constancia $(n=8)$, confianza en sí mismo $(n=4)$, y al entrenador, donde destacaban, el buen trabajo desarrollado por éste a nivel técnico-táctico $(n=8)$ y la preparación física $(n=6)$. Los fracasos deportivos, según los entrevistados, se debían, fundamentalmente, al escaso sacrificio del deportista $(n=8)$, a la falta de formación del entrenador $(n=5)$, y al poco apoyo económico recibido por las instituciones pertinentes $(n=3)$.

Pues bueno yo creo que hay gente que tiene muchas cualidades y muchas facultades, pero no llegan a explotarlas, lo han conseguido de manera fácil, pero no están dispuestas a apostar y dejar otros ámbitos de tu vida y meter "x" horas de entrenamiento (Sujeto 9, párrafo 65).

\section{Dimensión Psicológica}

Aquí se trataba de conocer si el deportista había trabajado con psicólogos y a qué aspectos psicológicos daban mayor importancia. Todos los entrevistados señalaron que los aspectos psicológicos tenían mucha importancia para llegar a la élite. El 95\% ( $n=19)$ coincidió en que, en alta competición, donde los niveles 
son tan similares, quien esté mejor psicológicamente en ese momento tiene más posibilidades de ganar. Además, consideraron clave ser fuerte psicológicamente para soportar las altas horas de duro entrenamiento y la presión a la que se ven sometidos en competiciones de máxima importancia. Los judokas destacan la perseverancia $(n=8)$, la constancia $(n=7)$, la capacidad de sacrificio $(n=6)$ y la autoconfianza $(n=5)$.

Soy muy constante, muy perseverante. Soy la constancia y la perseverancia, el estar cansada y seguir, el ponerme un objetivo e intentar conseguirlo (Sujeto 12, párrafo 30).

El entrenamiento de los aspectos psicológicos se llevó a cabo a través de psicólogos deportivos o del propio entrenador. En relación con las sensaciones que recuerdan en los campeonatos, 9 judokas señalan que presentaban mayor nerviosismo en los campeonatos a nivel nacional que a nivel internacional, por la presión de cosechar buenos resultados y clasificarse para las competiciones internacionales. En cuanto a las principales características psicológicas que debía tener un judoka de élite, los entrevistados destacaron la constancia $(n=10)$, la autoconfianza $(n=5)$ y la capacidad de sacrificio $(n=4)$.

\section{Dimensión Técnico-Táctica}

Con esta dimensión se quiso saber qué importancia daba el deportista a los aspectos técnico-tácticos, si consideraba que los había trabajado en su justa medida durante su formación deportiva y en qué etapa era necesario profundizar más en ellos. En este sentido, el $95 \%(n=19)$ de los entrevistados consideraron que el trabajo de la técnica era muy importante, pero, el $45 \%(n=9)$ de los judokas, opinaban que se debería comenzar a trabajar a partir de infantil 0 cadete, mientras que el $40 \%(n=8)$ pensaba que se tenía que desarrollar desde el inicio de la práctica deportiva.

Yo creo que, en el caso del judo, la técnica es la base del deporte, es como quién dice los cimientos desde los cuales luego ya puedes ir aprendiendo más cosas, si tú no tienes una buena base técnica es muy complicado poder aprender más cosas (Sujeto 2, párrafo 45).

El $80 \%(n=16)$ de los entrevistados estaban satisfechos con el trabajo técnico durante su formación deportiva, y aquéllos que no pensaban así $(n=4)$, estimaban que habían trabajado mucho los aspectos técnicos sin tener en cuenta la calidad de este trabajo. En cuanto a la importancia que otorgaban al trabajo táctico durante el proceso de formación deportiva, encontramos que el $100 \%$ $(n=20)$ de los sujetos le daban mucha importancia, sin embargo, muchos consideraban que se debía trabajar cuando el proceso de formación estuviera más avanzado, a partir de categoría de cadete $(n=8)$ o júnior $(n=7)$. Además, el $75 \%(n=15)$ de los entrevistados estaban satisfechos con el trabajo táctico llevado a cabo durante su formación deportiva. 


\section{Dimensión Condición Física}

Por último, en esta dimensión se estudia cómo han entrenado la condición física a lo largo de su formación deportiva, quién ha sido el responsable en su preparación, qué capacidades físicas consideran más importantes y cómo creen que se debería trabajar. De esta manera, el $60 \%(n=12)$ de los judokas señalaron que trabajo físico debía comenzar a partir de cadetes. Además, el $75 \%(n=15)$ de los deportistas olímpicos consideraban que se tenía que desarrollar integrada junto a la preparación técnico-táctica. También cabe destacar que, según el $90 \%$ $(n=18)$ de los sujetos, el responsable del trabajo de preparación física había sido el propio entrenador. En cuanto a las cualidades físicas fundamentales para llegar a ser un judoka de elite, los entrevistados consideraban que la fuerza $(n=14)$ y la resistencia $(n=14)$, ambas en sus distintas manifestaciones, eran las más importantes.

Pienso que se debe hacer una preparación física integrada, o sea, lo que es en el tatami con cuerdas, con kimonos, o sea, muy integrado en el tatami con aros y cosas de estas (Sujeto 11, párrafo 62). 

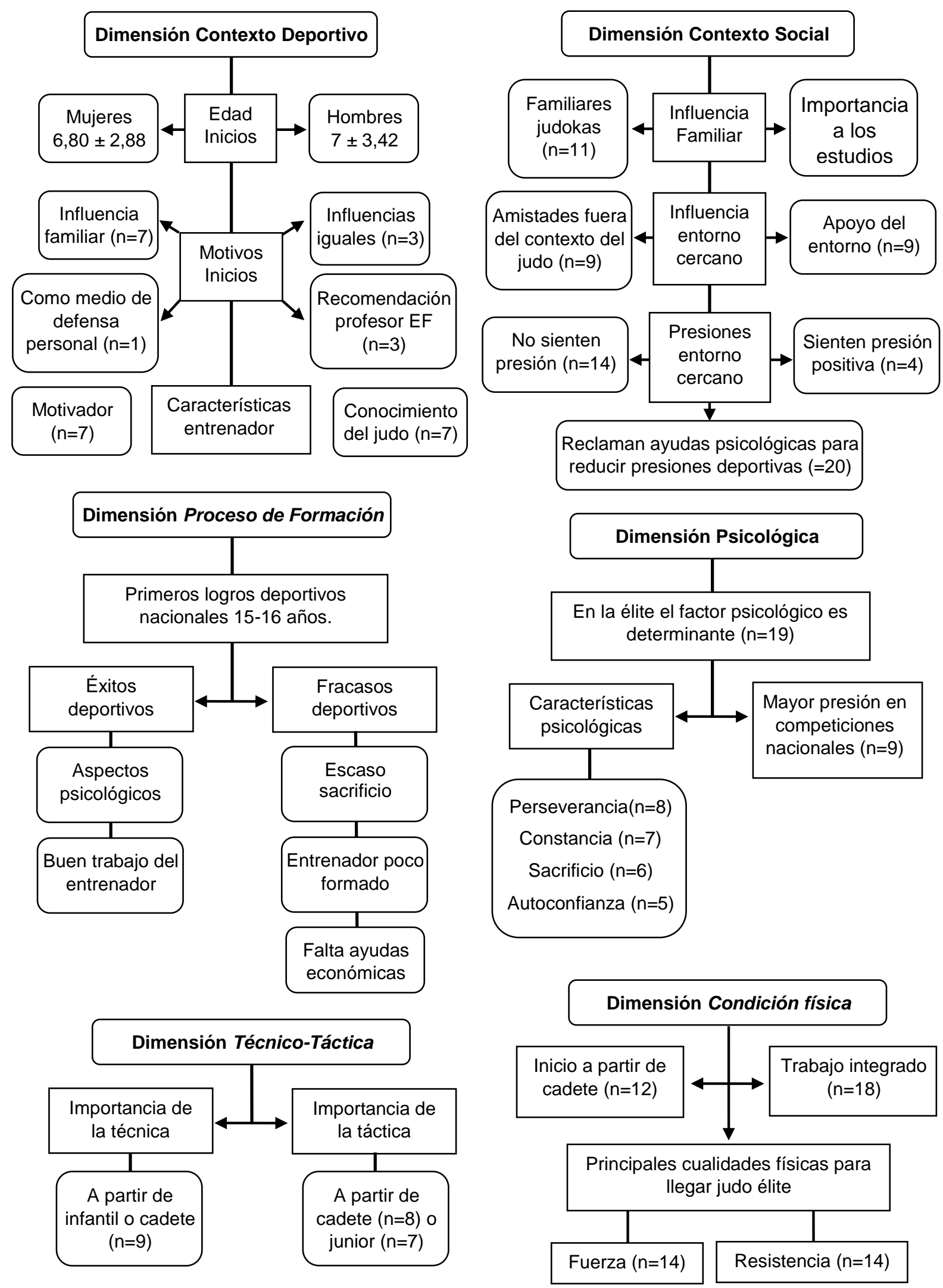

Figura 1. Factores que influyen en el proceso de formación de los judokas olímpicos. 


\section{DISCUSIÓN}

En el presente estudio se observa que los judokas olímpicos españoles se inician en la práctica deportiva en las edades comprendidas entre los 6-8 años, en consonancia con lo indicado por García, Campos, Lizaur y Pablo (2003). En esta iniciación, la familia es el principal factor que favorece el comienzo de esta práctica deportiva, ya que los miembros de la familia suelen ser modelos de gran alcance para los chicos y chicas (Sánchez-Miguel et al., 2013). Además, también influye el grupo de iguales o amigos, lo cual coincide con lo expresado por Lorenzo y Calleja (2010). Otro aspecto que ha ejercido influencia han sido las recomendaciones por parte de profesores de educación física, tal como han mostraron Moreno y Cervelló (2004). No obstante, para los judokas las personas más importantes de su entorno son sus padres y hermanos. Los padres normalmente ejercen la función primordial de introducir, alentar y mantener a sus hijos en la práctica deportiva (Amenabar, Sistiaga y García, 2008) proporcionándoles a su vez, las mejores condiciones para la enseñanza y la formación (Côté, Baker, y Abernethy, 2003). Esto, quizás en nuestra investigación se ve reforzado debido a que la mayoría de los entrevistados tienen algún familiar directo (padre, madre o hermano) medallista nacional o internacional en alguna disciplina deportiva, principalmente en Judo. Por otra parte, coincidiendo con otros estudios (Gimeno y Guedea, 2001; Weiss y Stuntz, 2004), los entrevistados señalan que el entrenador es una pieza importante de su entorno más cercano siendo su papel motivacional y de apoyo igual de importante que el de los padres. De igual forma, los amigos y compañeros son elementos importantes, debido a que las relaciones sociales implican una gran interacción social y emocional (Serra, Zaragoza y Generelo, 2014). Además, sienten que la ausencia de presión, por parte de la familia y de los entrenadores, es fundamental para el éxito deportivo, lo cual está en la línea de lo expresado por Anderson, Funk, Elliot, y Smith (2003). La mayoría de los judokas entrevistados dan gran relevancia a la formación académica, coincidiendo con lo expresado por Torregrosa, Sánchez y Cruz (2004), debido, fundamentalmente, a que consideran necesaria la formación académica por la importancia que ésta tiene para el desarrollo integral de la persona, y por las posibilidades laborales que los estudios le proporcionarán una vez terminado la etapa de alto rendimiento, lo cual concuerda con lo señalado por Álvarez, PérezJorge, González, y López (2014).

La mayoría de los judokas olímpicos empiezan a cosechar éxitos deportivos en la última fase de cadete y primera de júnior, es decir, entre los 1517 años. Esto coincide con investigaciones realizadas en diferentes deportes (Forteza, 2009; Leonard, 1996), donde la mayoría de los deportistas que llegan a la elite no han sido los mejores en las etapas inferiores, por lo que parece ser más beneficioso para el practicante retrasar la búsqueda del rendimiento hasta edades cercanas a la categoría sénior. En cuanto a la etapa de alta competición, los judokas olímpicos españoles comienzan a partir de los 17-18 años. En este sentido, Wylleman y Lavallee (2003) señalaron que aproximadamente a los 20 años se inicia la etapa de perfeccionamiento de los deportistas de elite. Estudios como los desarrollados por Gullich (2007), en deportistas Olímpicos en Atenas, y por Schumacher, Mroz, Mueller, Schmid y Ruecker (2006), sobre el proceso deportivo en ciclistas profesionales, constataron que la mayoría de los 
deportistas de elite comienza su etapa competitiva a nivel internacional en la categoría júnior e incluso sénior.

Los aspectos psicológicos son un factor determinante para los judokas olímpicos, quienes llegan a sopesar que en determinados momentos quien esté mejor psicológicamente tendrá más posibilidades de ganar. Además, consideran clave ser fuerte psicológicamente para soportar las numerosas horas de duro entrenamiento y la presión a la que se ven sometidos en competiciones de máxima importancia, lo cual ha sido considerado en diversos estudios (Abalde y Pino, 2016; Callister, Callister, y Staron, 1991; Ferreira, Gomes, Borges, Routen, y Almeida, 2015; Santos et al., 2015). En este sentido, los entrevistados aluden a la adquisición de determinados aspectos psicológicos individuales como claves para la consecución de resultados, entre los que destacan la perseverancia, la constancia, la capacidad de sacrificio y la autoconfianza, tal como fue señalado por Ferreira et al. (2015). Aunque casi todos los judokas han realizado de alguna manera un trabajo psicológico, no todos lo han hecho con un profesional de la psicología deportiva, sino que, en determinados casos, el propio entrenador es quien se ocupa de este tipo de trabajo, lo que hace necesario que él mismo adquiera nociones del entrenamiento psicológico, coincidiendo en este sentido con varios trabajos (Acosta, 2012; Capetillo, 2011; Contreras, Rojas y Palomino, 2012; Sava, y Ciuntea, 2013). Además, lo judokas destacan, como uno de los aspectos psicológicos más importantes, la motivación, ya que de ella va a depender en gran medida la continuidad del niño en la práctica deportiva o no, tal como señalan Piñar y Cárdenas (2010). Por otro lado, los deportistas entrevistados creen que el hecho de no saber gestionar la presión, de no tener altos niveles de autoconfianza y de motivación, es lo que impide a muchos judokas rendir al máximo y llegar a la elite deportiva. En este sentido, existen investigaciones que han demostrado que soportar situaciones potencialmente estresantes y saber gestionar la presión es garantía de éxito (Hernández, 2007).

Respecto a los elementos técnicos, se observa que los judokas entrevistados piensan que trabajar estos aspectos durante el proceso de formación es de máxima importancia, ya que consideran que la técnica es la base sobre la que construir el Judo una vez que te vas acercando a la etapa de alto rendimiento, lo cual coincide con expresado por Santos et al., (2015) y Osipov et al., (2016). En cuanto a los aspectos tácticos, más de la mitad de los judokas estiman que éstos son poco importantes durante el proceso de formación, adquiriendo mayor importancia conforme el judoka va creciendo y avanzando en las etapas de la formación deportiva, y considerando que se deben trabajar a partir de los 14-16 años. Sin embargo, numerosos autores piensan que en el Judo se debe trabajar a partir de la táctica desde las primeras etapas, ya que, a través de la misma se desarrolla la intuición, la anticipación y la percepción (Carratalá, 2000; Méndez, 1998; Molina y Castarlenas, 2002; Terrisse, 1996; Terrisse et al., 1995). En este sentido, Osipov et al. (2016) consideran que es necesario hacer hincapié en el proceso de preparación táctica de los judokas para conseguir éxito en las competiciones, lo cual no quiere decir que la enseñanza de la técnica se deje a un lado. De esta manera, Avelar y Figuereido (2009) señalan que el aprendizaje a través de la táctica necesita una base motriz técnica mínima que permita poder ejecutar las decisiones tácticas que se organizan sobre las habilidades motrices básicas, por lo que la 
preparación de ambos aspectos (técnica y táctica) resulta vital en la formación de judokas de elite (Osipov et al., 2016; Santos et al., 2015).

Por otro lado, los judokas entrevistados consideran que la condición física es importante durante el proceso de formación deportiva, pero que no es conveniente trabajarla desde la etapa de iniciación, sino a partir de los 14-16 años, coincidiendo con la etapa de desarrollo. Esto significa que debemos esperar a que el joven vaya alcanzando la madurez biológica y no ocasionar efectos adversos en los procesos relacionados con la adaptación a la actividad física, el crecimiento y la maduración (Díaz y Romero, 2007). En relación con la forma de entrenar la condición física durante el proceso de formación deportiva, todos los judokas dicen haberla entrenado de manera integrada junto a los aspectos técnico-tácticos, lo cual coincide con lo expresado por diferentes autores (Capetillo, 2011; Chirosa, Chirosa y Padial, 2010; Gallo, Gobbi y Ayala, 2013; Robles, 2003). Además, los entrevistados afirman que el responsable de la preparación física era, en todos los casos, el propio entrenador, y que empezaron a tener un preparador físico específico cuando estaban en la etapa de alta competición. Por otro lado, la mayoría de los entrevistados opinan que las cualidades físicas más importantes en los judokas de alta competición son la fuerza y la resistencia en sus diversas manifestaciones, lo cual está en relación con numerosos trabajos (Bonicht, 2007; García, 2004; Franchini et al., 2015), aunque no con la investigación de Pion et al. (2015) en la que los jóvenes judokas de elite destacan por su velocidad y agilidad.

\section{CONCLUSIONES}

El objetivo de este trabajo era conocer y analizar los itinerarios deportivos recorridos por los judokas españoles que habían representado a España en los Juegos Olímpicos celebrados en el siglo XXI. En este sentido, entre las conclusiones de este estudio destaca que los judokas olímpicos se iniciaron en la práctica deportiva alrededor de los siete años, principalmente por influencia familiar o de amigos. Además, los judokas empiezan a obtener resultados importantes cuando se acercan a la etapa de júnior, empezando a cosechar aquí sus primeras medallas nacionales e internacionales.

El entorno social más cercano resulta imprescindible para los judokas en las etapas de formación, lo cual también es relevante durante la etapa de alta competición. Entre las principales personas que los judokas consideran más importantes en su entorno social más cercano, están los padres y los hermanos, por ser el mayor apoyo emocional tanto en los buenos como en los malos momentos. Los entrenadores son fundamentales también por ser una fuente constante de motivación y mejora en el aspecto deportivo, así como los amigos como vía de escape en momentos de saturación física y psicológica. Además, los entrevistados consideran importante la formación académica, con vistas a un mayor enriquecimiento personal y a una futura inserción laboral. Por otro lado, para los judokas olímpicos es muy importante llevar una sincronización del entrenamiento técnico-táctico, la condición física y los aspectos psicológicos de forma integrada. También, los entrevistados piensan que las cualidades físicas más importantes son la fuerza y la resistencia en todas sus manifestaciones y que son determinantes la perseverancia, la capacidad de sacrificio y la 
constancia. También destaca el hecho de que los deportistas olímpicos consideran que, en general, durante las primeras etapas, los judokas que destacan son los más completos físicamente, pero que la obtención de resultados en edades tempranas no significa el éxito deportivo en categoría sénior. De esta manera, podemos concluir que el análisis del proceso de formación de los judokas olímpicos nos ha permitido conocer mejor los elementos más relevantes que han incidido en el logro de la excelencia deportiva.

Las principales limitaciones del estudio están relacionadas con la inexistencia de este tipo de estudios en Judo y con el modo en el que se llevó a cabo la entrevista. Muchos de los judokas ya se encontraban retirados y desvinculados del ámbito federativo, por lo que resultaba muy difícil encontrar un lugar físico donde estuvieran todos al mismo tiempo (competiciones, concentraciones...). A esto hay que añadir, el hecho de que algunos sujetos se encontraban viviendo fuera de España. Futuros estudios podrían dedicarse a analizar el proceso de formación de los judokas en los Juegos Olímpicos venideros y ver similitudes y diferencias con la investigación realizada. Además, se podría profundizar más a través de estudios de casos, entrevistando a sus familiares, amigos y entrenadores.

\section{REFERENCIAS}

Abalde, N., y Pino, R. (2016). Evaluación de la autoeficacia y de la autoestima en el rendimiento deportivo en Judo. Retos. Nuevas tendencias en Educación Física, Deporte y Recreación, 29, 109-113.

Acosta, E. (2012). Iniciación y Formación Deportiva: Una reflexión siempre oportuna. U.D.C.A. Actualidad y Divulgación Científica, 15, 57-65.

Álvarez, P. R., Pérez-Jorge, D., González, M. E., y López, D. (2014). La formación universitaria de deportistas de alto nivel: análisis de una compleja relación entre estudios y deporte. Retos. Nuevas tendencias en Educación Física, Deporte y Recreación, 26, 94-100.

Amenabar, B., Sistiaga, J. J., y García, E. (2008). Revisión de los distintos aspectos de la influencia de los padres y las madres en la práctica de la actividad física y deporte. Apunts: Educación Física y deportes, 93, 29-35.

Anderson, J. C., Funk, J. B., Elliot, R., y Smith, P. H. (2003). Parental support and pressure and children's extracurricular activities: relationships with amount of involvement and affective experience of participation. Journal of Applied Developmental Psychology, 24, 241-257.

Anguera, M. T. (2008). Evaluación de programas desde la metodología cualitativa. Acción Psicológica, 5(2), 87-101.

Avelar, B., y Figuereido, A. (2009). La iniciación a los deportes de combate: interpretación de la estructura del fenómeno lúdico luctatorio. Revista de Artes Marciales Asiáticas, 4(3), 44-57.

Baker, J., Horton, S., Roberston-Wilson, J., y Wall, M. (2003). Nurturing sport expertise: Factors influencing the development of elite athlete. Journal of Sport Sciences and Medicine, 2, 1-9.

Bonicht, J. (2007). Evolución de la fuerza muscular en el tren superior en sucesivos combates de judo. Universidad de Granada. 
Callister, R., Callister, R. J., y Staron, R. S. (1991). Physiological chracteristics of elite judo athletes. International Journal Sport Medicine, 12, 106-203.

Capetillo, R. (2011). Factores sociales que influyen en la formación deportiva de adolescentes. Revista Lúdica Pedagógica, 16(2), 39-48.

Carratalá, V. (2000). La iniciación al judo. III Jornadas internacionales de Judo. Málaga: Unisport.

Chirosa, L. J., Chirosa, I. J., y Padial, P. (2000). Efecto del entrenamiento integrado sobre la mejora de la fuerza de impulsión en un lanzamiento en suspensión en balonmano. Revista Motricidad, 6, 155-174.

Cohen, J. (1960). A coefficient of agreement for nominals scales. Educational and Psychological Measurement, 20, 37-46.

Côté, J., Baker, J., y Abernethy, B. (2003). From play to practice: A developmental framework for the acquisition of expertise in team sport. En J. Starkes y K. A. Ericsson (Eds.), Recent Advances in Research on Sport Expertise (pp. 89-114). Champaign: Human Kinetics.

Díaz, P., y Romero, R. (2007). Deporte de Alto Rendimiento. Gerencia, ciencia y tecnología. Tunja: Indeportes Boyacá.

Ferreira, T., Gomes, J. C., Borges, H., Routen, A., y Almeida, A. (2015). Elite coaches' views on factors contributing to excellence in orienteering. Cultura, Ciencia y Deporte, 10, 77-86.

Forteza, A. (2009). Treinamento Desportivo. Carga, estrutura e planejamento (2 ${ }^{\mathrm{a}}$ ed.). São Paulo: Editorial Phorte.

Franchini, E., Del Vecchio, F. B., Ferreira, U. y Candau, R. (2015). Specificity of performance adaptations to a periodized judo training program. Revista Andaluza de Medicina del Deporte, 8(2), 67-72.

Gallo, L., Gobbi, S., y Ayala, C. (2013). Parâmetros e princípios da programação de Exercício Físico. En F. Gomes, S. Gobbi, J. Costa y L. Gobbi (Eds.), Exercício Físico no Envelhecimento Saudável e Patológico. Curitiba: Editora CRV.

García, J. M. (2004). Análisis diferencial entre los paradigmas experto-novatos en el contexto del alto rendimiento deportivo en judo. Tesis Doctoral. Universidad de Castilla la Mancha, Toledo.

García, J. M., Campos, J., Lizaur, P., y Pablo, C. (2003). El talento deportivo. Formación de élites deportivas. Madrid: Gymnos.

García-Moya, I., Moreno, C., Rivera, F., Ramos, P., y Jiménez-Iglesias, A. (2011). Iguales, familia y participación en actividades deportivas organizadas durante la adolescencia. Revista de Psicología del Deporte, 21(1), 153-158.

Giménez, F. J. (2003). La formación del entrenador en la iniciación al baloncesto.

Sevilla: Wanceulen.

Gimeno, F., Buceta, J. M., y Pérez-Llantada, M. C. (2007). Influencia de las variables psicología en el deporte de competición: evaluación mediante el cuestionario. Características psicología relacionadas con el rendimiento deportivo. Psicothema, 19(4), 667-672.

Gimeno, F., y Guedea, J. A. (2001). Evaluación e intervención psicológica en la «promoción» de talentos deportivos en judo. Revista de Psicología del deporte, 10(1), 103-125.

Goetz, J. P., y Lecompte, M. D. (1984). Etnography and qualitative desing in educational research. Orlando (Florida): Academic Press, inc. 
Güllich, A. (2007) Training - Support - Success: Control-related assumptions and empirical findings. Saarbruücken: University of the Saarland.

Hernández, J. G. (2007). Herramientas aplicadas al desarrollo de la concentración en el alto rendimiento deportivo. Cuadernos de Psicología del Deporte, 7(1), 61-70.

Landis, J. R., y Koch, G. G. (1977). The measurement of observer agreement for categorical data. Biometrics, 33(1), 159-174.

Leonard, W. M. (1996). The odds of transiting from one level of sports participation to another. Sociology of Sport Journal, 13, 288-299.

Lorenzo, A. (2001). Hacia un nuevo enfoque del concepto de talento deportivo. Revista de Entrenamiento Deportivo, 15(2), 27-33.

Lorenzo, A., y Calleja, J. (2010). Factores condicionantes del desarrollo deportivo. Bizkaia: Diputación Foral de Bizkaia. Dirección General de Deportes.

Lorenzo, A., y Sampaio, J. (2005). Reflexiones sobre los factores que pueden condicionar el desarrollo de los deportistas de alto nivel. Apunts. Educación Física y Deportes, 80(2), 63-70.

MacNamara, A., Button, A., y Collins, D. (2010). The role of psychological characteristics in falicitating the pathway to elite performance part 1 : Identifying mental skills and behaviors. The Sport Psychologist, 24, 52-73.

Mahamud, J., Tuero, C., y Márquez, S. (2005). Características psicológicas relacionadas con el rendimiento: comparación entre los requerimientos de los entrenadores y la percepción de los deportistas. Revista de Psicología del Deporte, 14(2), 237-251.

Méndez, A. (1998). El pensamiento táctico en el judo: un proceso generado desde la etapa inicial mediante el juego. Lecturas: Educación Física y Deportes, 11.

Molina, J., y Castarlenas, J. (2002). Bases para una propuesta para la enseñanza del Judo en el contexto escolar. En J. Castarlenas y J. Molina (Eds.), El Judo en la Educación Física Escolar (pp. 29-44). Barcelona: Hispano Europea.

Moreno, J. A., y Cervelló, E. (2004). Influencia de la actitud del profesor en el pensamiento del alumno hacia la educación física. Revista Internacional de Ciencias Sociales y Humanidades SOCIOTAM, 14(1), 33-51.

Mujika, I. (2010). Intense training: the key to optimal performance before and during the taper. Scandinavian Journal of Medicine \& Science in Sports, 2, 24-31.

Osipov, A., Kudryavtsev, M., Struchkov, V., Kuzmin, V., Bliznevsky, A., y Plotnikova, I. (2016). Expert analysis of the competitive level of Young russian judo athletes who train for active attack fighting. Journal of Physical Education and Sport, 16(4), 1153-1158. DOI:10.7752/jpes.2016.04185

Palao, J. M., Ortega, E., Calderón, A., y Abraldes, J. A. (2008). Características del proceso de formación deportiva en el atletismo español. Estudio descriptivo Campeonatos de España de la Juventud (años 1997 y 2003). Murcia: Diego Marín.

Pazo, C. L. (2010). El proceso de formación de los jugadores españoles de fútbol de alta competición. Tesis Doctoral. Universidad de Huelva.

Piñar, M. I., y Cárdenas, D. (2010). La competición como herramienta formativa. Diferentes propuestas en minibasket. Wanceulen E.F. Digital, 7. 
Pion, J., Segers, V., Fransen, J., Debuyck, G., Deprez, D., Haerens, L., ...Lenoir, M. (2015). Generic anthropometric and performance characteristics among elite adolescent boys in nine different sports. European Journal of Sport Science, 15(5), 357-366. http://dx.doi.org/10.1080/17461391.2014.944875

Regnier, G. M., Salmela, J. H., y Russell, S. T. (1993). Talent detection and development of sport. En R. N. Singer, M. Murphey, y L. K. Tennant (Eds.), Handbook of Research on Sport Psychology. New York: MacMillan.

Robles, A., Robles, J., Giménez, F. J., y Abad, M. T. (2016). Validación de una entrevista para estudiar el proceso formativo de judokas de élite. Revista Internacional de Medicina y Ciencias de la Actividad Física y del Deporte, 16(64), 723-738. Doi: http://dx.doi.org/10.15366/rimcafd2016.64.007

Robles, J. (2003). La enseñanza del judo mediante una metodología activa. Una propuesta de entrenamiento integrado. Lecturas: Educación Física $y$ deportes. Revista Digital, 64.

Ruiz, G., y Salinero, J. J. (2011). El entrenador de alto nivel en triatlón: entorno próximo y cualidades fundamentales para el rendimiento. International Journal of Sport Science, 23(7), 113-125.

Ruiz, R. (2007). Características de liderazgo en el deporte del judo. Revista de Psicología del Deporte, 16(1), 9-24.

Ruiz, R. (2008). Aportaciones del análisis subdimensional del cuestionario de personalidad BFQ para la predicción del rendimiento en judokas jóvenes de competición. Cuadernos de Psicología del Deporte, 8(1), 5-29.

Sáenz-López, P., Giménez, F. J., Sierra, A., Sánchez, M., Ibáñez, S. y Pérez de Rueda, R. (2006). La formación del jugador de baloncesto de alta competición. Huelva: Wanceulen.

Sánchez-Miguel, P. A., Leo, F. M., Sánchez-Oliva, D., Amado, D., y GarcíaCalvo, T. (2013). The Importance of Parents' Behavior in their Children's Enjoyment and Motivation in Sports. Journal of Human Kinectics, 36, 169177.

Santos, L., Fernández-Río, J., Almansba, S., Sterkowicz, S., y Callan, M. (2015). Perceptions of top-level Judo coaches training and performance. International Journal of Sports \& Coaching, 10(1), 145-158.

Sava, M. A., y Ciuntea, M. L. (2013). Study on the opinion of romanian coaches regarding the psychological factor in judo training. Sport \& Society, 13, 7882.

Schumacher, Y. O., Mroz, R., Mueller, P., Schmid, A., y Ruecker, G. (2006) Succes in elite cycling: A prospective and retrospective analysis of race results. Journal of Sports Sciences, 24(11), 1149-1156.

Serra, J. R., Zaragoza, J., y Generelo, E. (2014). Influencias de "otros significativos" para la práctica de actividad física en adolescentes. Revista Internacional de Medicina y Ciencias de la Actividad Física y el Deporte, 14(56), 735-753.

Singer, R. N., y Janelle, C. M. (1999). Determining sport expertise: From Genes to Supremes. International Journal of Sport Psychology, 30, 117-150.

Terrisse, A. (1996). Analyse de la transposition didactique du Judo: evolution du 'savoir combattre' dans l'enseignement du Judo à l'école à travers la Revue EPS de 1950 a 1993. Revue Française de Pédagogie, 116, 65-76.

Terrisse, A., Quesada, Y., Sauvegrain, J., y Hiegel, P. (1995). Le Savoir Combattre: Essai d'Élucidation. Revue EPS, 252, 26-29. 
Thomas, J. R., y Nelson, J. K. (2007). Métodos de investigación en actividad física. Barcelona: Paidotribo.

Torregrosa, M., Sánchez, X., y Cruz, J. (2004). El papel del psicólogo del deporte en el asesoramiento académico - vocacional del deportista de elite. Revista de Psicología del Deporte, 13(2), 215-228.

Weiss, M. R., y Stuntz, C. P. (2004). A little friendly competition: peer relationships and psychosocial development in youth sport and physical activity contexts. En M. R. Weiss (Ed.), Developmental Sport and Exercise Psychology: a lifespan perspective (pp. 35-66). Morgantown: FIT.

Wylleman, P., y Lavallee, D. (2003). A developmental perspective on transitions faced by athletes. En M. Weiss (Ed.), Developmental Sport Psychology. Morgantwon, WV: Fitness Information Techonology.

Referencias totales / Total references: 64 (100\%)

Referencias propias de la revista / Journal's own references: 2 (3,1\%)

Rev.int.med.cienc.act.fís.deporte - vol. 19 - número 74 - ISSN: 1577-0354 